\title{
Drogenmissbrauch - eine Herausforderung für die Kinder- und Jugendpsychiatrie
}

\section{Schoor \\ C. Möller}

\author{
Drug Abuse - a Challenge for Child and Adolescent Psychiatry
}

\section{Zusammenfassung}

Die Schnittstellenproblematik der herkömmlichen Drogenhilfe ist durch die Trennung von Entzug und Therapie mit einer hohen Rückfallquote belastet. Früh traumatisierte Jugendliche erleben wiederholte Beziehungsbrüche, wie sie dies aus ihrer Lebensgeschichte kennen. Auf der Therapiestation Teen Spirit Island haben wir ein Konzept entwickelt mit maximaler Konstanz der therapeutischen Beziehungen von der Aufnahmephase (Entzug und Motivation) über die Behandlung der zugrunde liegenden seelischen Störung bis zur Jugendhilfe mit integrierter Nachsorge. Dieses Behandlungsmodell verfolgt das Ziel, dass der drogenabhängige Jugendliche in den Versorgungsstrukturen besser aufgehoben ist.

Schlliisselwörter

Drogenmissbrauch · Jugendpsychiatrie · Teen Spirit Island

\section{Abstract}

The lack of continuity in the traditional treatment of substance abusers is extremely problematic due to the high relapse rate. Youth traumatised at young age experience a recurrant severance of relationships which they have previously been exposed to. At our therapy ward TEEN SPIRIT ISLAND (TSI) we have developed a concept which focuses on the establishment of a good therapeutical contact from admission (withdrawel and motivation) via the treatment of underlying mental disturbance to the postclinical care.

\section{Key words}

Drugabuse · Adolecentpsychiatry · Teen Spirit Island
Drogenabhängige Jugendliche drohen durch das Netzwerk des psychosozialen Betreuungsangebots zu fallen. Die traditionelle Drogenhilfe hat ihren Schwerpunkt in der Betreuung von Erwachsenen. In der stationären Jugendhilfe ist der Drogenkonsum häufig ein Ausschlusskriterium. Die Behandlung drogenkonsumierender Jugendlicher stößt in der allgemeinen Kinder- und Jugendpsychiatrie schnell an ihre Grenzen. Vor fünf Jahren wurde in Hannover die Therapiestation für drogenabhängige Kinder und Jugendliche Teen Spirit Island (TSI) in Kooperation mit der Drogenberatungsstelle Prisma und der stationären Jugendhilfeeinrichtung Stepkids realisiert $[2-4,6]$.
Auf der Therapiestation für drogenabhängige Kinder und Jugendliche Teen Spirit Island ist neben der qualifizierten Entgiftung eine längerfristige stationäre psychiatrisch-psychotherapeutische Behandlung der zugrunde liegenden seelischen Störung, die ihre Wurzeln in einem gestörten Entwicklungsprozess hat, möglich. Die Station ist Teil der Kinder- und Jugendpsychiatrie des Kinderkrankenhauses auf der Bult in Hannover. Sie hat 12 Behandlungsplätze, 4 in der Aufnahmephase und 8 in der Behandlungsphase. Nach einer 4-8 Wochen dauernden Entgiftungs- und Motivationsphase wechselt der Jugendliche in die Behandlungsphase, wo der Schwerpunkt die psychiatrische psychotherapeutische Behandlung der zugrunde liegenden see-

Institutsangaben

Kinderkrankenhaus auf der Bult, Hannover

Korrespondenzadresse

Dr. med. Christoph Möller · Dr. med. Christian Schoor · Kinderkrankenhaus auf der Bult .

Janusz-Korczak-Allee 12 30173 Hannover

Bibliografie

Suchttherapie 2005; 6: 28 - 30 ๑ Georg Thieme Verlag KG Stuttgart · New York

DOI 10.1055/s-2005-858016

ISSN 1439-9903 


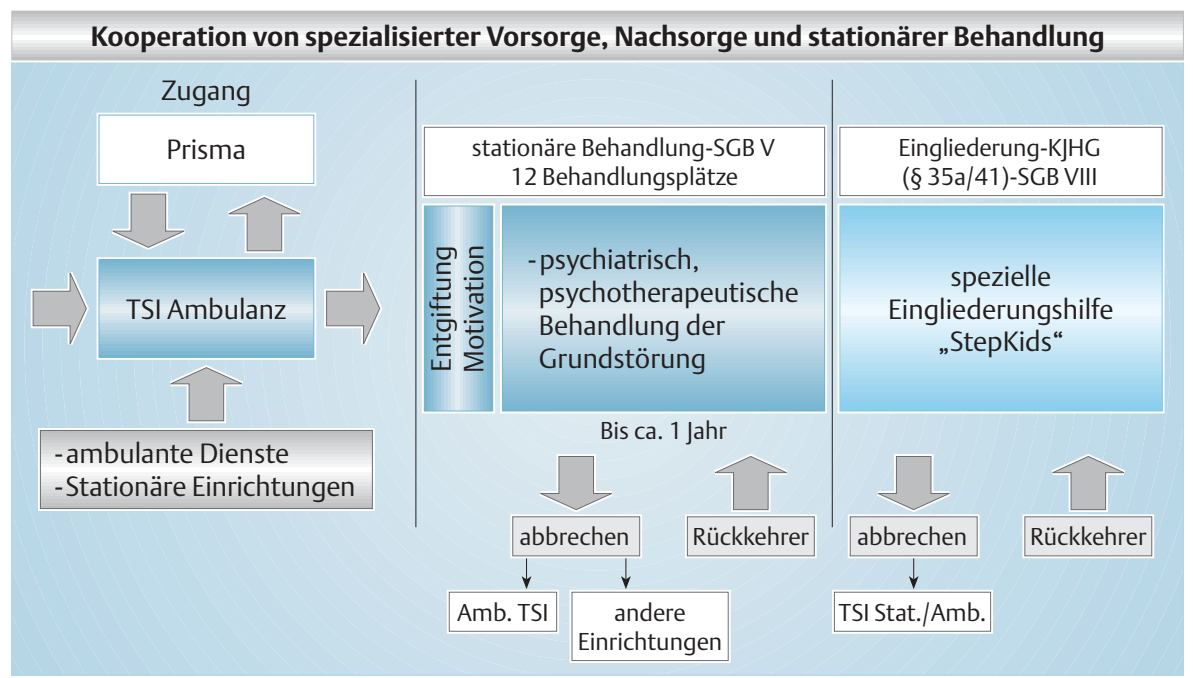

Abb. 1 Kooperationsnetzwerk von Teen Spirit Island.

lischen Störung ist. Hier ist ein Verbleib im Einzelfall bis zu 10 Monaten möglich [5].

Behandelt werden Jugendliche im Alter von 12 bis 18 Jahren. Der Tagesablauf ist zeitlich und inhaltlich klar strukturiert. In den Alltags- und Therapiegruppen soll Verbindlichkeit im Umgang miteinander und mit den Erwachsenen erarbeitet werden. Das Reden über sich selbst ist im Jugendalter noch ungeübt, oft beängstigend und bedrohlich. Die traumatischen Erfahrungen werden häufig vom unmittelbaren Erleben abgespalten. Oft sind diese Erfahrungen nicht als Geschichte verfügbar und deshalb nicht erzählbar. Vor allem in der Anfangszeit auf Station leiden die Jugendlichen unter ihrer Sprachlosigkeit in Bezug auf ihre Erlebniswelt und ihre Lebensgeschichte $[2,5]$.

Im Rahmen des stationären Settings wird häufig reinszeniert, was erfahren wurde, in Worten aber nicht auszudrücken ist. Auf Station kann nach Bedingungen gesucht werden, die helfen, aus den Spannungszuständen herauszufinden, die eine gewisse Sicherheit und Ruhe vermitteln und zur Stabilisierung beitragen. Ich-Fähigkeiten können entwickelt und trainiert werden, nach Interessen gesucht und Ressourcen aufgebaut werden.

In den gruppen- und einzeltherapeutischen Sitzungen können die der Drogensucht zugrunde liegenden Konflikte aufgedeckt und bearbeitet werden. In kleinen Schritten können Worte oder künstlerische Ausdrucksformen erarbeitet werden, die traumatische Reinszenierung als Ausdrucksform tritt in den Hintergrund. Auf die Drogen kann längerfristig verzichtet werden, wenn die erlebten Traumata bearbeitet wurden und Kraftquellen erschlossen sind. Die Gruppe kann bei diesem Prozess als stabilisierend und haltbringend erlebt werden.

Mit TSI wurde ein spezielles kinder- und jugendpsychiatrisches Angebot entwickelt in enger Zusammenarbeit mit der Drogenberatungsstelle Prisma und der stationären Jugendhilfeeinrichtung Stepkids. Prisma hat einen Schwerpunkt in der Betreuung drogenabhängiger Jugendlicher und der Präventionsarbeit. Eine Betreuung nach $\S 35 \mathrm{a}$ und $\S 41$ des Kinder- und Jugendhilfegesetzes (KJHG) ist bei Prisma möglich. In den Räumlichkeiten von Prisma findet regelmäßig eine kinder- und jugendpsychiatrische Sprechstunde für drogenkonsumierende und abhängige Jugendliche und ihre Angehörigen statt. Gemeinsame Gespräche mit Mitarbeitern beider Einrichtungen sind möglich. Die Vermittlung bei speziellen Fragestellungen ist kurz und unbürokratisch. Nach Beendigung der Therapie auf TSI ist eine Überleitung zu Stepkids möglich, die sich auf Jugendliche mit einer Drogenproblematik als Eingliederungshilfe nach $\S 35$ a des KJHG spezialisiert hat [4]. Bei Stepkids sind der Wiedereinstieg in die Schule, begleitende Berufsausbildung und weitere Verselbstständigung inhaltliche Ziele. Bei Rückfällen ist eine kurzfristige Wiederaufnahme zur Krisenintervention auf TSI möglich. Gemeinsame Freizeitangebote der beiden Einrichtungen erleichtern die Eingewöhnung. Die ambulante Nachbetreuung findet bei Bedarf über die Ambulanz von TSI statt. Eine Weiterbetreuung durch Prisma ist möglich [2].

In der traditionellen Drogenhilfe erfährt das Beziehungsangebot durch die institutionelle Trennung von Drogenentzug und Entwöhnung/Rehabilitation einen Bruch (so genannte Schnittstellenproblematik). Das vorhandene Misstrauen und die innere Emigration der Jugendlichen werden verstärkt und es kommt häufig zu Rückfällen. Demgegenüber haben wir auf der Therapiestation TSI ein Konzept entwickelt mit maximaler Konstanz in den therapeutischen und betreuenden Beziehungen von der Aufnahmephase (Drogenentzug und Motivation) über die Behandlung der zugrunde liegenden seelischen Störung (Behandlungsphase) bis zur stationären und ambulanten Nachsorge. Im Einzelfall kann der Jugendliche von der ambulanten Phase über die stationäre Therapie bis zur Wiedereingliederungshilfe betreut und behandelt werden. Dieses Modell bietet eine hohe Beziehungskontinuität und eine bedarfsgerechte, unbürokratische Zusammenarbeit unterschiedlich spezialisierter, sich ergänzender Einrichtungen [3, 4]. Das zentrale Element der therapeutischen und pädagogischen Arbeit ist die intensive persönliche Beziehung. Weiterhin ist durch Öffentlichkeitsarbeit einer bestehenden Pauschalisierung und Stigmatisierung der Sucht als selbstverschuldete Erkrankung entgegenzutreten. Diese bestehende Diskriminierung drogenabhängiger Menschen steht einer Integration, einem „Haltfinden“ in den Familien und in der Gesellschaft, entgegen [1].

\section{Literatur}

${ }^{1}$ Gaebel W, Zäske H, Baumann A. Stigma erschwert Behandlung und Integration. Dtsch Arztbl 2004; 101 (48): A 3253 - 3255 
2 Möller C. JUGEND SUCHT. Esslingen: Gesundheitspflege initiativ, 2003 ${ }^{3}$ Möller C. Stationäre und ambulante Therapieangebote für Drogenabhängige Jugendliche. In: Möller C (Hrsg). Drogenmißbrauch im Jugendalter, Ursachen und Wirkungen. Göttingen: Vandenhoeck \& Ruprecht, 2005

${ }^{4}$ Möller C. Kooperationsnetzwerk für drogenabhängige Jugendliche. Forum SOZIAL 2004; 3: $18-20$
${ }^{5}$ Möller C, Thoms E. Teen Spirit Island. Eine Therapiestation für drogenabhängige Kinder und Jugendliche stellt sich vor. Systhema 2001; 15 (1): $39-45$

${ }^{6}$ Thoms E. Therapiestation für drogenabhängige Kinder und Jugendliche TEEN SPIRIT ISLAND. SuchtReport 1999; 6: 39-43 